\title{
Factores determinantes en la viabilidad del turismo de negocios en Puebla, México, como oportunidad de desarrollo económico
}

\author{
Determining factors in the viability of business \\ tourism in Puebla, Mexico as an opportunity for \\ economic development
}

Ramón Sebastián A. ${ }^{1}$, Abigail Claudio M. ${ }^{2}$, Emigdio Larios G. ${ }^{3}$

Resumen: El turismo de negocios comprende el conjunto de corrientes turísticas cuyo motivo de viaje está vinculado a la realización de actividades profesionales y laborales. La problemática fundamental se ve reflejada en el asentamiento y crecimiento de grandes empresas nacionales e internacionales en el sector industrial y de servicios. El objetivo general de este trabajo fue determinar cómo los cinco factores de viabilidad económica del turismo son una oportunidad de desarrollo económico en el turismo de negocios en la ciudad de Puebla, México. La metodología se llevó a cabo mediante un enfoque cuantitativo utilizando los métodos deductivo, analítico y sintético. Las técnicas de investigación requeridas fueron la encuesta y la entrevista. Los resultados del coeficiente de correlación de Pearson fueron de 0,47 (sector empresarial), 0,43 (infraestructura), 0,41 (servicios turísticos), 0,36 (tecnología) y 0,32 (políticas públicas). La principal conclusión sugiere que hay evidencia a favor de la viabilidad de la ciudad de Puebla en el turismo de negocios y como oportunidad de desarrollo económico.

Palabras clave: Turismo de negocios, viabilidad económica, desarrollo económico.
Abstract: Business tourism includes the set of tourist flows where the reason for the trip is linked to professional and work-related activities. The fundamental issue is reflected in the establishment and growth of large national and international companies in the industrial and services sectors. The general aim of this study was to determine how the five factors of economic viability in tourism are an opportunity for economic development in business tourism in the city of Puebla, México. The methodology was carried out using a quantitative approach with deductive, analytical and synthetic methods. The research techniques required were the survey and the interview. The results of Pearson's correlation coefficient were 0.47 (business sector), 0.43 (infrastructure), 0.41 (tourism services), 0.36 (technology) and 0.32 (public policies). The main conclusion suggests that there is evidence in favor of the viability of Puebla for business tourism and as an opportunity for economic development.

Key words: Business tourism, economic viability, economic development.

(Presentado: 25 de abril de 2017. Aceptado: 30 de mayo de 2017)

\footnotetext{
${ }^{1}$ Facultad de Administración, Benemérita Universidad Autónoma de Puebla, México. E-mail: raclemxayahoo.com.mx

${ }^{2}$ Benemérita Universidad Autónoma de Puebla, México. E-mail: abyclaudio8̨agmail.com

${ }^{3}$ Benemérita Universidad Autónoma de Puebla, México. E-mail: herr.lariosqgmail.com
} 


\section{INTRODUCCIÓN}

Las reuniones de negocios detonan inversión e infraestructura impactando de manera importante en los destinos en donde se llevan a cabo, demostrando así capacidad para atraer inversiones de diferentes rubros, su principal beneficio es aumentar la ocupación hotelera del destino. Se entiende por turismo de negocios al conjunto de corrientes turísticas cuyo motivo de viaje está vinculado con la realización de actividades laborales y profesionales, llevadas a cabo en reuniones de negocios de diferentes propósitos y magnitudes (Sistema Integral de Información de Mercados Turísticos, 2015).

En los últimos años el turismo de negocios en México ha ido creciendo y retomando su verdadera importancia en la economía, por lo cual el país ocupa el quinto lugar en turismo de reuniones en América con 163 eventos, después de Estados Unidos, Brasil, Canadá y Argentina. Este segmento genera una derrama económica anual de 32 mil 500 millones de dólares, de los cuales $56 \%$ es gasto en empresas turísticas y el resto en diferentes sectores de la economía, logrando la generación de 151.100 empleos, de los cuales 85.000 son directos y 66.000 indirectos. Además, México ocupa el lugar número 22 en el ranking de eventos internacionales de la Asociación Internacional de Congresos y Convenciones. Por otra parte, la ciudad de Puebla es considerada como una de las más importantes de México destacando en los contextos histórico, demográfico, cultural e industrial, posicionándose como la puerta de entrada hacia el sureste mexicano.

\section{Turismo de negocios}

México es uno de los líderes del turismo internacional receptivo, con más de veinte millones de turistas ingresados por año a partir de los inicios del siglo XXI. Esta posición de privilegio se fundamenta en una variada oferta de productos turísticos, desde el clásico turismo de sol y playa en varios centros vacacionales localizados en ambas costas del país, el turismo arqueológico revalorizando el patrimonio, el turismo alternativo con fuerte vinculación con la naturaleza, hasta el turismo de negocios.

Se entiende por turismo de negocios al conjunto de corrientes turísticas cuyo motivo de viaje está vinculado con la realización de actividades laborales y profesionales llevadas a cabo en reuniones de negocios de diferentes propósitos y magnitudes (Sistema Integral de Información de
Mercados Turísticos, 2015). También comprende viajes de vinculación entre instituciones, por ejemplo académicas y viajeros que asisten a congresos, convenciones, ferias y exhibiciones, en función de intereses de determinados sectores de afinidad cultural, social, política o económica más identificable por la modalidad del encuentro que por la especificidad del objetivo. En otros países se conoce como turismo MICE, cuyas siglas en ingles son: Meetings, Incentives, Conventions and Exhibitions (Reuniones, Incentivos, Conferencias y Exposiciones). México anteriormente era conocido como turismo de negocios, pero evolucionó al término "turismo de reuniones", por considerar que abarca mejor el conjunto de actividades que se realizan. Aunque en general hace referencia al turismo de negocios, no todas las actividades conllevan este propósito. De acuerdo al Consejo de Promoción Turística (2016) el turismo de negocios se puede clasificar en:

- $\quad$ Congresos de asociaciones: Es el encuentro de grandes grupos de personas donde se discute o intercambian puntos de vista acerca de un tema en común, el cual puede ser profesional, cultural, deportivo, académico, entre otros. Pueden tener una duración de varios días y con sesiones simultáneas. Estas reuniones no son orientadas a los negocios.

- $\quad$ Ferias y exposiciones comerciales: Son eventos comerciales o culturales que reúnen a miembros de un sector empresarial, profesional o social, organizado con el propósito de dar a conocer bienes o servicios y llevar a cabo labores de relaciones públicas o de comercialización. Los asistentes acuden con el propósito principal de visitar el área de exposición.

- Viajes de incentivo: Son una estrategia moderna gerencial enfocada en reconocer a las personas que alcanzaron o excedieron objetivos comúnmente relacionados a las ventas o productividad, dirigida a los participantes que demuestran un mejor desempeño en su trabajo.

- $\quad$ Reuniones corporativas: Son reuniones grupales normalmente patrocinadas por una compañía, donde los asistentes son parte de la misma o una extensión de ella (clientes, proveedores, socios). Muchas veces los gastos corren por parte de la misma corporación, puesto que es obligatorio asistir. 
De la anterior clasificación esta investigación se centró en el turismo de negocios que comprenden ferias, exposiciones comerciales y reuniones corporativas o de negocios. El turismo de negocios presenta claras ventajas sobre otras modalidades, tales como la planificación a detalle, contratación con gran antelación, los eventos se realizan durante temporadas generalmente medias y bajas, rompe la estacionalidad, y lo disfrutan colectivos de poder adquisitivo alto o medio alto, entre otras. Este puede ser individual cuando la persona se desplaza y visita un destino turístico por motivos de trabajo, también puede ser grupal cuando se tiene la posibilidad de seleccionar el destino turístico en el cual realizaran un congreso, expo industrial, convención o negocios con la condición de que el lugar cumpla con los requisitos para la organización (COBACH SONORA, 2015).

La situación actual y estabilidad económica que ha vivido México en los últimos cinco años, así como el crecimiento económico han provocado que la industria de las reuniones crezca. En este sentido se percibe que las reuniones corporativas, así como las ferias y exposiciones son los tipos de reuniones que mayor crecimiento han presentado. Además, México ocupa el lugar número 22 en el ranking mundial de eventos internacionales por parte del ICCA (Internacional Congress and Convention Association, 2015). En el 2014 se realizaron 266.117 reuniones, de las cuales 182.300 (69\%) fueron reuniones corporativas o de negocios, 33.800 congresos de asociaciones (13\%), 7.661 (3\%) programas de incentivos, 6.440 exposiciones (2\%) y el resto otro tipo de eventos (Consejo de Promoción Turística, 2016).

De acuerdo a la revista internacional especializada en turismo de negocios The Meeting Planner's Magazine (2016), Puebla ocupa el séptimo lugar en la lista de las diez mejores ciudades para hacer negocios en México. De acuerdo a la Secretaria de Turismo del Estado de Puebla en el reporte del perfil del visitante, y en el que se refleja el motivo del viaje, se percibe la problemática de estudio, en donde el principal motivo de viaje sigue siendo por descanso o placer $55,3 \%$ contra la posición baja de negocios o trabajo con un $6.5 \%$ de un total de 13 millones 98 mil visitantes (Secretaría de Turismo, 2015).

\section{Puebla industrial}

Según cifras del Consejo Nacional de Población (CONAPO) el Estado de Puebla actualmente cuenta con una población de 6.254.597 habitantes, convirtiéndolo en el quin- to más poblado del país (CONAPO, 2016). Además posee templos, conventos, una situación geografía privilegiada e historia regional, por lo que es reconocida mundialmente como "Relicario de América" (VisitMéxico, 2016) y recientemente ha sido nombrada "Ciudad Creativa del Diseño" por la Organización de las Naciones Unidas para la Educación, la Ciencia y la Cultura (UNESCO) en la categoría de diseño, al fomentar el potencial creativo, social y económico de las colectividades locales para promover la diversidad cultural.

En el ámbito fabril, experimentó una industrialización temprana que arrancó en los años treinta del siglo XIX con la producción mecanizada de textiles de algodón, debido al establecimiento de estas fábricas en la ribera del río Atoyac, abarcando Puebla y Tlaxcala, creando el corredor industrial del Atoyac. Así, a partir de 1897, este se encontraba conformado por 13 fábricas textiles: La Covadonga, La María, La Constancia, La Economía, La Beneficencia, El Patriotismo, La Independencia, San Juan Bautista Amatlán, El Molino de Enmedio y Mayorazgo en Puebla y las otras tres restantes en Tlaxcala. Además de su cercanía y relación económica con el centro de la ciudad, compartían el aprovechamiento del río Atoyac como la fuente de energía para su funcionamiento gracias al establecimiento de presas, infraestructura hidráulica e incluso plantas de energía hidroeléctrica que garantizaban el continuo funcionamiento de la maquinaria industrial a partir de la corriente fluvial. Este sector fue el más importante dentro de la estructura económica e industrial del estado por más de 100 años, hasta 1965, seguido de los sectores alimentos y bebidas debido al número de establecimientos, inversión y valor de la producción (Rosas, 2013).

Puebla es considerada la puerta de entrada del sureste mexicano, al ser una vitrina en donde todos los bienes y servicios están a la vista. Desde su fundación hace 486 años y debido a su ubicación geográfica que le permite ser enlace entre la parte centro y este de México, ha sido un estado estratégico para el comercio entre Europa y América. Además, según datos del Instituto Nacional de Estadística y Geografía (INEGI) goza de la estabilidad económica mexicana y ser una de las ciudades mejor calificadas en seguridad pública (INEGI, 2016), así como ser punto de atracción de inversión por parte de empresas extranjeras con estándares internacionales. Puebla dispone de una infraestructura especializada para recibir y exponer productos industriales, llevar a cabo congresos, exposiciones, 
y convenciones de grandes magnitudes, entre los que destacan el Auditorio del Complejo Cultural Universitario (CCU), Auditorio Acrópolis Puebla, Auditorio Metropolitano, Teatro Principal, Teatro de la Ciudad, Centro de Convenciones Puebla William O. Jenkins, Centro Expositor y de Convenciones de Puebla y el World Trade Center Puebla.

Recientemente ha sido calificada como excelente destino para Inversión Extranjera Directa por empresas como Standard \& Poor's y Fitch Ratings, su capacidad laboral es competitiva y especializada en industria y servicios, ya que destaca por ser una ciudad educativa (Sistema Nacional de Información Estadística Educativa - SNIEE, 2014). Según la Secretaría de Educación Pública (SEP) Puebla ofrece una amplia oferta de instituciones académicas, tanto públicas como privadas a nivel superior por lo que se posiciona como el primer estado con mayor número de instituciones de educación superior (SEP, 2017).
En los últimos años los sectores estratégicos en el estado de Puebla son automotriz y autopartes, metalmecánica, química, plásticos, textil-confección, muebles, agroindustrial, alimentos frescos y procesados, turismo, artículos de decoración, mármol, minería, servicios médicos y las tecnologías de la información, posicionándolo como una de las más importantes en estos rubros en el territorio nacional. Cabe mencionar que las principales empresas extranjeras establecidas en el Estado son alemanas, brasileñas, canadienses, suizas, francesas, y estadounidenses (Área de Libre Comercio de las Américas - ALCA, 2005).

En el rubro de infraestructura productiva, es el segundo productor automotriz en México, sólo después de Aguascalientes. Actualmente el Estado cuenta con 18 parques industriales y/o tecnológicos (Secretaría de Economía, 2016) reflejando su importancia y magnitud industrial (Tabla 1).

\section{TABLA 1. Parques industriales de la ciudad de Puebla.}

\begin{tabular}{ll}
\hline Parques Industriales & \\
Área Industrial San Felipe Chachapa & Parque Industrial EI Carmen \\
Conjunto Industrial Chachapa & Parque Industrial Esperanza \\
Corredor Industrial Cuatlancingo, A.C. & Parque Industrial Tehueya \\
Fraccionamiento Industrial Resurrección & Parque Industrial Textil \\
Parque Industrial 5 de mayo & Parque Industrial Valle de Tehuacán \\
Parque Industrial Volkswagen, Fraccionadora & FINSA, Puebla Industrial Park \\
Industrial del Norte, S.A. de C.V. (FINSA) & \\
Parque Industrial Puebla 2000 & CIT del ITESM \\
Parque Industrial San Miguel, Corredor Industrial QuetzalcóatI & IDIT de la Universidad IBERO Puebla \\
Área Uno Corredor Industrial Quetzalcóatl & INAOE
\end{tabular}

Fuente: Secretaría de Economía (2016).

Hoy en día el Estado ha tenido un gran desarrollo industrial ya que según el Sexto Informe de Gobierno del gobernador Rafael Moreno Valle, la inversión directa en este rubro ha sido de 5.145 millones de dólares y se han creado 125.795 empleos formales (El Economista, 2017).

A inicios de 2016 Puebla aparece como la quinta entidad con más empresas en México, al contabilizar 303.233. Según el Directorio Estadístico Nacional de Unidades Económicas (DENUE) fueron Audi, Taiwán, Volkswagen, Cemex, Cruz Azul, Danús, Liverpool e IVER las empresas más representativas en cuanto a inversión en lo últimos años (INEGI, 2016). Es por esto que Puebla es considerada una ciudad importante en el sector industrial por la cantidad de empresas existentes dentro de los parques industriales y la facilidad que ofrece para la realización de negocios.

\section{Desarrollo económico}

Frecuentemente los conceptos de desarrollo y crecimiento económico se usan de forma intercambiable, aunque fijan su atención en cuestiones distintas. El crecimiento económico se refiere a la elevación de los niveles de ingreso de un país, mientras que desarrollo económico está ligado al concepto de libertad para ser y actuar, estar libre de hambre, enfermedad, represión, ignorancia, y pobreza, de forma que las personas puedan desarrollar sus capacidades. Aunque diferentes, ambos conceptos están fuertemente vinculados, no es posible tener desarrollo eco- 
nómico sin crecimiento económico. La estrecha relación entre desarrollo y crecimiento económico implica que resolver el enigma del crecimiento económico es fundamental para entender cómo impulsar el desarrollo.

El desarrollo es un concepto que ha ido evolucionando, por lo que no tiene una definición única. El desarrollo económico de una sociedad implica un aumento a largo plazo de la capacidad para proveer a la población de bienes económicos cada vez más diversificados, se basa en el adelanto de la tecnología y los ajustes institucionales e ideológicos que tal adelanto exige. En tal sentido, no basta con la generación de riqueza, con un mejor uso de los elementos que promueven la producción y que optimizan el uso de las mejoras tecnológicas que se generan en las sociedades, sino que se requiere convertir esa mejora en accesibilidad a mejores formas de vida para el conjunto de la población (Kuznets, 1955). Castillo (2011) define el desarrollo económico como el proceso en virtud del cual la renta real per cápita de un país aumenta durante un largo período de tiempo. En otros términos, el desarrollo es un proceso que implica la expansión continua del potencial económico e incrementos sucesivos en las condiciones de vida de todas las personas o familias de un país o comunidad. Se entiende por desarrollo económico de un país el crecimiento sostenido en su magnitud como unidad económica y como la capacidad de producir y obtener riqueza, además éste puede ser tanto a nivel del desarrollo personal como aplicado también a países o regiones. Ya sea en uno o en otro caso el desarrollo está ligado al sustento y la expansión económica de modo tal que garantice el bienestar, se mantenga la prosperidad y satisfaga las necesidades personales o sociales y con ello la dignidad humana. A la inversa, el estancamiento y la decadencia pueden definirse como la incapacidad sostenida de crecimiento de la magnitud económica de la nación, o su persistente disminución. El desarrollo económico surge con el advenimiento del modo de producción capitalista, el cual implica un aumento en las tasas de ganancia que permiten el proceso de acumulación del capital. Estos saltos de acumulación cuantitativa son causados por varios factores que se pueden combinar, tales como reducción de costos, incorporación de técnicas o máquinas más productivas, y sobreexplotación de las fuerzas de trabajo. No sólo hay factores internos sino también externos, por ejemplo, en el caso del desarrollo económico de una nación, el contexto internacional de tasas de ganancias elevadas o las inversiones externas. El pensamiento racional económico de políticas públicas apunta a sostener el nivel de crecimiento, para ello existen regulaciones institucionales llevadas a cabo con el fin de incentivar y fomentar la eficiencia del sistema productivo.

Con todas estas premisas, se puede concluir que el enfoque de desarrollo univariable (sólo económico) es un concepto anacrónico; por lo que, si se analizan los aspectos más comunes y distintos de estos conceptos, se puede afirmar que el desarrollo está comprendido en cuatro ejes fundamentales:

- $\quad$ Económico: El desarrollo sin recursos y sin inversiones, como la infraestructura o el equipamiento, no se podrá generar ya que estas condiciones de integración permiten que los procesos se financien concibiendo así la base material del desarrollo.

- Social. Para generar desarrollo es necesario lograr que la población se incorpore y haga suyos los procesos de desarrollo. Sin embargo, en la práctica, estos esfuerzos se han circunscrito a los temas materiales (como hospitales y escuelas) cuando existe la necesidad de generar capacidades intrínsecas a las personas como son la educación o la salud y otras condiciones de integración a la sociedad.

- Ambiental: La calidad y el uso sostenible de los recursos son una condición necesaria para el desarrollo. No puede concebirse como desarrollada una sociedad que ha perdido o está en proceso de pérdida de su capital natural en aras de crear condiciones económicas temporales.

- Institucional: Requieren capacidades para su desarrollo económico, social, ambiental e institucional. Todos estos procesos son desarrollados por personas, y son las personas las que necesitan no sólo conocer conceptos, sino también "saber cómo" generar estos desarrollos.

Esta investigación se basa en el eje económico, el cual comprende los recursos económicos, inversiones e infraestructura para que se creen y desarrollen más empresas industriales y de servicios en la ciudad de Puebla. El objetivo general fue determinar cómo los cinco factores de viabilidad económica del turismo son una oportunidad de desarrollo económico en el turismo de negocios en la ciudad de Puebla. La hipótesis general fue de tipo correlacional siendo enunciada; "La ciudad de Puebla es viable para desarrollar el turismo de negocios como oportunidad 
de desarrollo económico". Se fijaron cinco hipótesis de trabajo englobando el sector empresarial, infraestructura, servicios turísticos, políticas públicas y tecnología, siendo éstas directamente proporcionales para la viabilidad del turismo de negocios. Además, el estudio contribuyó con aportaciones teóricas, metodológicas y empresariales dentro del contexto del turismo.

\section{METODOLOGÍA}

Esta investigación realizada bajo un enfoque económico-turístico, reflejando la problemática en el asentamiento y crecimiento de grandes empresas nacionales e internacionales en los ramos industrial y de servicio en la ciudad de Puebla. La investigación se realizó mediante un enfoque cuantitativo utilizando los métodos: deductivo, analítico y sintético. Las técnicas de investigación requeridas fueron la encuesta y la entrevista, utilizando un cuestionario con 20 ítems con opción de respuesta en puntos Likert de $1=$ totalmente en desacuerdo a 5 = totalmente de acuerdo. Para probar la confiabilidad de la escala se usó el coeficiente Alfa de Cronbach, obteniendo un valor de 0,857 que cumple el criterio de "bueno" para su aplicación.
Las unidades de análisis fueron los 7 hoteles más representativos en la categoría business class, ubicados en la periferia del parque industrial FINSA Autopista México Puebla km 117. Los sujetos de estudio seleccionados fueron los turistas de negocios que visitaron la ciudad de Puebla durante el mes de febrero del 2017, siendo aproximadamente 85.600 turistas de negocios (Secretaria de Cultura y Turismo, 2015).

Para determinar el tamaño de la muestra se utilizaron los criterios de $95 \%$ de confianza y $5 \%$ de error, $50 \%$ de probabilidad a favor y $50 \%$ de probabilidad en contra, resultando 384 turistas de negocios, y ajustándose a 390 encuestas. Se utilizaron 6 encuestadores - estudiantes de la licenciatura de Administración Turística de la Benemérita Universidad Autónoma de Puebla, con una cuota personal de 65 encuestas. Se diseñó un modelo de viabilidad económica híbrido, que sirvió como referencia para determinar las variables independientes y sus constructos. Dicho modelo está conformado por cinco variables (sector empresarial, infraestructura, servicios turísticos, políticas públicas y tecnología). En la Tabla 2 se aprecian los constructos de las cinco variables antes mencionadas.

TABLA 2. Constructos de las variables incluidas en el cuestionario.

\begin{tabular}{|c|c|c|c|}
\hline Dimensión & Definición & Variable & Ítem \\
\hline Infraestructura & $\begin{array}{l}\text { Es el conjunto de obras y } \\
\text { servicios que permiten, } \\
\text { en general, el desarrollo } \\
\text { socioeconómico de un país } \\
\text { y que el turismo utiliza para } \\
\text { impulsar su actividad. }\end{array}$ & $\begin{array}{l}\text { 1. Accesibilidad } \\
\text { 2. Centros expositores } \\
\text { 3. Ubicación geográfica } \\
\text { 4. Servicios públicos }\end{array}$ & $\begin{array}{l}\text { 1. La accesibilidad para trasladarse a } \\
\text { su destino en Puebla es eficiente. } \\
\text { 2. Los centros expositores de Puebla } \\
\text { tienen la capacidad para albergar } \\
\text { grandes eventos de negocios. } \\
\text { 3. La ubicación geográfica de Puebla } \\
\text { permite por las conexiones con } \\
\text { puntos estratégicos hacer negocios. } \\
\text { 4. La existencia de los servicios } \\
\text { públicos a los que tuvo acceso } \\
\text { fueron de calidad. }\end{array}$ \\
\hline
\end{tabular}




\begin{tabular}{llll}
\hline Dimensión & Definición & Variable & Ítem \\
\hline Servicios turísticos & Los servicios turísticos son & 1. Hoteles & 1. Los hoteles dedicados a los \\
& el conjunto de realizaciones, & 2. Restaurantes & negocios en Puebla cumplen con sus \\
& hechos y actividades, & 3. Atracción turística & expectativas. \\
& tendientes a producir & 4. Prestador de & 2. Los restaurantes ofrecen un espacio \\
prestaciones personales que & servicios turísticos & adaptado para realizar negocios. \\
& satisfagan las necesidades & & 3. Encontró sitios en Puebla que le son \\
& del turista y contribuyan & & atractivos y cumplen con sus intereses. \\
& al logro de facilitación, & & 4. El destino le facilitó la prestación \\
& acercamiento, uso y disfrute & & de servicios adicionales tales como \\
& de los bienes turísticos. & & traductores, enfermeras, entre otros, \\
& & & en caso de solicitarlo.
\end{tabular}

Políticas Públicas

\section{Conjunto de acciones} intencionales y causales, orientadas a la realización de un objetivo de interés o beneficio público, en correspondencia con el cumplimiento de funciones públicas (Aguilar y Lima, 2014).

\section{Seguridad.}

2. Políticas públicas nacionales.

3. Políticas públicas internacionales.

4. Incentivos gubernamentales.
1. La seguridad pública de Puebla permite su libre permanencia.

2. Realizar trámites en Puebla le es sencillo para cerrar un trato empresarial. 3. Los acuerdos internacionales que se implementan en México, ayudan a la inversión en Puebla.

4. El gobierno le proporcionó facilidades en algún trámite requerido para sus negociaciones.
Tecnología
La totalidad de los métodos a los que se ha llegado racionalmente y que tienen una eficacia absoluta en todos los campos de la actividad humana.
1. Innovación.

2. Publicidad de redes.

3. Personal.

4. Disponibilidad de herramientas tecnológicas.
1. Durante su estadía los equipos tecnológicos que se emplearon fueron innovadores al cumplir con sus expectativas.

2. La información en internet acerca de los servicios en Puebla fue completa. 3. El personal que lo atendió en su estancia en Puebla está capacitado para el manejo de equipos tecnológicos. 4. Las herramientas tecnológicas que solicitó durante su estadía en Puebla estuvieron a su disponibilidad. 
Posteriormente se procedió a recolectar y vaciar la información obtenida en un archivo de Excel para su análisis estadístico, utilizando el coeficiente de correlación de Pearson que sirvió para medir la magnitud de relación de manera cuanti- tativa entre las variables relacionadas. Para la interpretación de los resultados del coeficiente de correlación de Pearson se utilizó la siguiente escala numérica de interpretación y nivel de significancia de los resultados. Ver la Tabla 3.

TABLA 3. Interpretación de la escala de coeficiente de correlación de Pearson.

\begin{tabular}{lll}
\hline Valor del coeficiente & Interpretación & Símbolo \\
\hline 0,00 & Correlación nula & $*$ \\
0,01 a 0,19 & Correlación positiva muy baja & $* *$ \\
0,2 a 0,39 & Correlación positiva baja & $* * *$ \\
0,4 a 0,69 & Correlación positiva moderada & $* * * *$ \\
0,7 a 0,89 & Correlación positiva alta & $* * * * *$ \\
0,9 a 0,99 & Correlación positiva muy alta & $* * * * *$ \\
1,00 & Correlación positiva grande y perfecta & \\
\hline
\end{tabular}

Fuente: (Goviden, 1985).

\section{RESULTADOS}

De acuerdo a las hipótesis de trabajo establecidas se presentan los resultados obtenidos en la Tabla 4.

TABLA 4. Hipótesis de trabajo y resultados del coeficiente de correlación de Pearson (r).

\begin{tabular}{|c|c|c|}
\hline Hipótesis & Pearson ( $r$ ) & Comentarios \\
\hline H1: Sector empresarial & 0,47 & $\begin{array}{l}\text { La hipótesis sometida a prueba, se considera que aporta evidencia } \\
\text { a favor, siendo una variable directamente proporcional para la } \\
\text { viabilidad de la ciudad de Puebla en el turismo de negocios siendo } \\
\text { así una oportunidad de desarrollo económico. La correlación } \\
\text { es positiva y moderada. }\end{array}$ \\
\hline
\end{tabular}

H2: Infraestructura

0,43
La hipótesis sometida a prueba, se considera que aporta evidencia a favor, siendo una variable directamente proporcional para la viabilidad de la ciudad de Puebla en el turismo de negocios siendo así una oportunidad de desarrollo económico.

La correlación es positiva y moderada.
0,41

0,32
La hipótesis sometida a prueba, se considera que aporta evidencia a favor, siendo una variable directamente proporcional para la viabilidad de la ciudad de Puebla en el turismo de negocios siendo así una oportunidad de desarrollo económico. La correlación es positiva y moderada.
La hipótesis sometida a prueba, se considera que aporta evidencia a favor, siendo una variable directamente proporcional para la viabilidad de la ciudad de Puebla en el turismo de negocios siendo así una oportunidad de desarrollo económico. La correlación es positiva y baja. 
H5: Tecnología

0,36
La hipótesis sometida a prueba, se considera que aporta evidencia
a favor, siendo una variable directamente proporcional para la
viabilidad de la ciudad de Puebla en el turismo de negocios siendo
así una oportunidad de desarrollo económico. La correlación
es positiva y baja.

\section{CONCLUSIONES}

La presente investigación logró alcanzar el objetivo y comprobar la hipótesis general y las de trabajo, a partir del modelo hibrido de viabilidad económica propuesto, conformado por cinco variables independientes. Se concluye que éstas aportan evidencia a favor al ser positivas y directamente proporcionales a la viabilidad de la ciudad de Puebla en el turismo de negocios y como oportunidad de desarrollo económico al correlacionarse estadísticamente y de forma cuantitativa a través del coeficiente de correlación de Pearson. Se muestra que las variables independientes sector empresarial con un valor de 0,47 de coeficiente de correlación de Pearson, la variable infraestructura con un valor de 0,43 y la variable servicios turísticos con un valor de 0,41 son las que más relación e influencia tuvieron, seguidas de las variables independientes tecnología con un valor de 0,36 y por último la variable políticas públicas con un valor de 0,32. A manera de resumen se determina que estas cinco variables son factores de viabilidad para el turismo de negocios de la ciudad de Puebla y su desarrollo económico, ya que en conjunto oscilan de manera positiva entre los rangos moderado y bajo. Así, la principal conclusión sugiere que hay evidencia a favor de la viabilidad de la ciudad de Puebla en el turismo de negocios y como oportunidad de desarrollo económico. Por último, la investigación fue original y específica, aportando al conocimiento teórico conceptos sobre turismo de negocios, Puebla industrial y desarrollo económico. Así como metodológicamente la aportación de un modelo híbrido de viabilidad que permitió la correlación de las variables en juego. Es importante indicar que los resultados obtenidos sólo corresponden a su aplicación en la ciudad de Puebla en el mes de febrero del 2017, por lo que se recomienda en un futuro realizar otras investigaciones en diferentes temporadas del año.

\section{REFERENCIAS}

Aguilar, C., \& Lima, M. (2014). ¿Qué son y para qué sirven las políticas públicas? Obtenido de http://secretariageneral.univalle.edu.co/consejoademico/ temasdediscusion/2014/Documentos_de_interes_general/Lecturas_politica_publica/Que\%20 son\%20y\%20para\%20que\%20sirven\%20las\%20 politicas\%20publicas Aguilar.pdf

ALCA. (2005). Puebla, México Ciudad Candidata para la Secretaría Permanente del Área de Libre Comercio de las Américas. Obtenido de http://www.ftaa-alca. org/tnc/submissions/tni152p2 s.pdf

Castilo, P. (2011). Política económica: Crecimiento económico, desarrollo económico, desarrollo sostenible. Revista Internacional del Mundo Económico y del Derecho III, 1-12.

COBACH SONORA. (2015). Servixios Turísticos Capacitación del Trabajo.

CONAPO. (2016). Consejo Nacional de Población. Obtenido de http://www.conapo.gob.mx/es/CONAPO/Proyecciones_Datos

Consejo de Promoción Turística. (2016). La relevancia económica de las reuniones en México 2016. Obtenido de http://www.siimt.com/work/ models/siimt/Resource/41f9c173-1c38-49f2bbcd-9aa3a43417ea/2016_REReuniones_EstudioFINAL.pdf

El Economista. (15 de enero de 2017). IED destaca en Puebla durante gestión de Moreno Valle. Obtenido de http://eleconomista.com.mx/estados/2017/01/15/ied-destaca-puebla-durante-gestion-moreno-valle

El Universal. (2015). Puebla, ciudad ideal para universitarios.

Obtenido de http://www.unionpuebla.mx/ar- 
ticulo/2015/02/17/educacion/puebla-ciudad-ideal-para-universitarios

Goviden, L. (1985). Introducción a la Estadística. Colombia: McGraw Hill.

Hernández, R., Fernández, C., \& Baptist, P. (2006). Metodología de la Investigación. México: McGraw Hill.

INEGI. (2016). Directorio Estadístico Nacional de Unidades Económicas.

Obtenido de http://www.beta.inegi.org.mx/app/ mapa/denue/

INEGI. (abril de 2016). Encuesta Nacional de Seguridad Pública Urbana (ENSU).

Obtenido de http://www.inegi.org.mx/saladeprensa/boletines/2016/ensu/ensu2016 04.pdf

Internacional Congress and Convention Association . (2015). ICCA Statistics Report 2015.

Obtenido de file:///C:/Users/Aby\%20Claudio\%20 Morales/Downloads/ICCA\%20Statistics\%20Report 2015.pdf

Kuznets, S. (1955). Economic rrowth and income inequality. The American Economic Review 45, 1, 1-28.

Rosas, S. (noviembre de 2013). Agua e industria en Puebla. El establecimiento de la fábrica textil La Covadonga, 1889-1897. Relaciones (Zamora) 34, 136.

Secretaria de Cultura y Turismo. (2015). Perfil de visitante Puebla 2015. Recuperado el 29 de 03 de 2017, de http://turismo.puebla.gob.mx/images/ estadisticas/Perfil-de-visitante-2015-2.pdf

Secretaría de Turismo. (2015). Perfil del Visitante, Destino: Puebla, Puebla. Obtenido de http://turismo.
puebla.gob.mx/images/estadisticas/Perfil-de-visitante-2015-2.pdf

Secretaría de Economía. (2016). Información Económica y Estatal Puebla.

Obtenido de https://www.gob.mx/cms/uploads/ attachment/file/85638/puebla.pdf

SEP. (marzo de 2017). Universidades por Estado. Obtenido de http://www.sep.gob.mx/es/sep1/ sep1_Educacion Superior\#.WPpIB k1 IV

Sistema Integral de Información de Mercados Turísticos . (2015). Glosario de términos utilizados para la promoción del turismo.

Obtenido de http://www.siimt.com/work/models/ siimt/Resource/5e20adab-d5b4-4434-996e-5af4d4c6e90e/PDF Glosario2.pdf

SNIEE. (2014). Sistema de Información Estadística Educativa. Obtenido de http:// www.snie.sep.gob.mx/ descargas/estadistica_e_in $\urcorner$ dicadores/estadistica_e_indicadores_educati-vos_21PUE.pdf

The Meeting Planner's Magazine. (26 de octubre de 2016). Top 10 Convention \& Exhibition Centers Mexico. Obtenido de http://www.mdcmagazine.com/index.php?option=com_k2\&view=item \&id=1314\%3Atop-10\%E $2 \% 80 \% A C$-convention-exhibition-centers-mexico-2016\&ltemid=66\&limitstart=1

VisitMéxico. (2016). Puebla, Ciudad Patromonio. Obtenido de http://www.visitmexico.com/es/ciudad-pa-trimonio- 